\title{
Harvesting triplet excitons for application in polymer solar cells
}

\author{
M. Arif, ${ }^{1}$ K. Yang, ${ }^{1}$ L. Li, ${ }^{1}$ P. Yu, ${ }^{1}$ S. Guha, ${ }^{1, a)}$ S. Gangopadhyay, ${ }^{2}$ M. Förster, ${ }^{3}$ and \\ U. Scherf ${ }^{3}$ \\ ${ }^{1}$ Department of Physics and Astronomy, University of Missouri, Columbia, Missouri 65211, USA \\ ${ }^{2}$ Department of Electrical Engineering, University of Missouri, Columbia, Missouri 65211, USA \\ ${ }^{3}$ Makromolekulare Chemie, Bergische Universität Wuppertal, Wuppertal 42119, Germany
}

(Received 23 September 2008; accepted 23 January 2009; published online 13 February 2009)

\begin{abstract}
Triplet enhanced ladder-type poly (para-phenylene) polymer (PhLPPP) with covalently bound trace amounts of palladium blended with a fullerene derivative [[6,6]-phenyl $\mathrm{C}_{61}$-butyric acid methyl ester (PCBM)] shows power conversion efficiencies (PCE) almost ten times greater than with pristine ladder-type polymer (with no palladium atom) blended with PCBM. The steady state optical properties of the triplet and nontriplet-enhanced polymers are comparable; the enhanced PCE and external quantum efficiency in PhLPPP photovoltaics are attributed to the presence of long-lived mobile triplet excitons. Furthermore, the luminescence from PhLPPP blends measured in a delayed setup correlates very well with the efficiency of the solar cells. (C) 2009 American Institute of Physics. [DOI: 10.1063/1.3082081]
\end{abstract}

Interfacial dissociation of triplet excitons constitutes another mechanism for enhancing photovoltaic (PV) efficiencies in polymer heterojunction-based solar cells, which are now becoming viable options for solar panels. The PV process in organic materials stems from the generation of singlet excitons upon efficient photon absorption, which may further result in a spin-flip leading to the formation of triplet excitons. Triplet excitons show strong spin exchange interaction and long life times compared to the singlet manifold, which aids their diffusion process toward donor-acceptor junctions, enhancing the PV process in organic solar cells. ${ }^{1}$

Great improvements in power conversion efficiencies of polymer solar cells, which are typically based on phase segregated blends of polymer semiconductors and fullerene derivatives have been recently observed. Such improvements entail development of low bandgap ${ }^{2,3}$ and organometallic polymers, ${ }^{4,5}$ tandem cell architectures, ${ }^{6}$ and controlling interfacial separations to match exciton diffusion lengths (few tens of nanometers). ${ }^{7}$ A common bulk heterojunction system which gives power efficiencies in the 4\%-5\% range consists of poly(3-hexylthiophene) (P3HT) and [6,6]-phenyl $\mathrm{C}_{61}$-butyric acid methyl ester (PCBM) ${ }^{8,9}$ Theoretical predictions show that donor polymers should have a bandgap energy below $1.74 \mathrm{eV}$ to achieve power efficiencies of $10 \% .^{10}$ This has resulted in a number of approaches to tailor the bandgap of polymers. In parallel, the synthesis and use of organometallic donor materials for efficient conversion of solar energy have been on the rise. Many of these materials are based on small organic molecules with a combination of phosphorescent dye molecules with iridium or platinum complexes. ${ }^{6,11}$

The central idea behind the inclusion of heavy metal atoms in the polymer backbone for PV applications is that it allows transitions between the singlet and triplet manifolds. ${ }^{12,13}$ A low concentration of metal atoms enables strong localized spin-orbit coupling. Introducing metal atoms in organic materials is typically achieved in two ways: by dispersing heavy metal atoms into the organic material or by

\footnotetext{
a) Author to whom correspondence should be addressed. Electronic mail: guhas@missouri.edu.
}

chemically attaching the heavy metal atoms to the organic molecules and polymers. The latter involves attaching metal atoms such as platinum or palladium to the polymer backbone usually by a side chain sequence during the synthesis process.

The polymer used in this study, is a diphenyl-substituted ladder-type poly(para-phenylene) (PhLPPP) containing a trace concentration of covalently bound $\mathrm{Pd}$ atoms. Trace quantities of Pd atoms in PhLPPP open up an efficient decay channel for migrating triplet excitons. Additionally, the basic photophysics [photoluminescence (PL) and absorption] of this polymer is comparable to its counterpart with no $\mathrm{Pd}$ atoms methylated-LPPP (MeLPPP). We note that the Pd contents (as estimated by inductively coupled plasma optical emission spectroscopy) are $<5$ ppm for MeLPPP and 120 $200 \mathrm{ppm}$ for PhLPP, respectively. ${ }^{14} \mathrm{PhLPPP}$ has served as an important system for elucidating fundamental properties of electrophosphorescence ${ }^{15}$ and temperature-dependent triplet diffusion. ${ }^{14}$ Rather than using different synthetic routes for metallated polymers, our results show that existing synthetic routes where only a trace concentration of metal is used as the catalyst during polymerization results in high efficiency polymer solar cells.

The solar cells were fabricated using PhLPPP or MeLPPP as the electron donor and PCBM as the electron acceptor material. Indium tin oxide spin-coated with poly(3,4-ethylene-dioxythiophene)/poly(styrene sulphonate) (PEDOT-PSS) served as the hole collection electrode and $\mathrm{Ca} / \mathrm{Al}$ served as the electron collecting electrode. PhLPPP:PCBM and MeLPPP:PCBM blends were prepared in different weight ratios, namely, 2:1, 1:1, and $1: 2$ by dissolving the polymers in chlorobenzene. The optimal concentration and spin speeds resulted in film thicknesses of $60-80 \mathrm{~nm}$, as verified by a step profilometer and a spectroscopic ellipsometer (JA Woollam V-Vase). The thickness of the PEDOT-PSS layer was $\sim 70 \mathrm{~nm}$. All fabrication steps were carried out in a $\mathrm{N}_{2}$ glove-box (MBraun) with less than $1 \mathrm{ppm}$ of $\mathrm{O}_{2}$ and $\mathrm{H}_{2} \mathrm{O}$.

Device efficiencies were measured in air mass (AM) 1.5 $\mathrm{G}$ illumination condition with the light source operating at $100 \mathrm{~mW} / \mathrm{cm}^{2}$ (Oriel 96000, $150 \mathrm{~W}$ solar simulator). The current-voltage measurements were carried out by a Keithley 

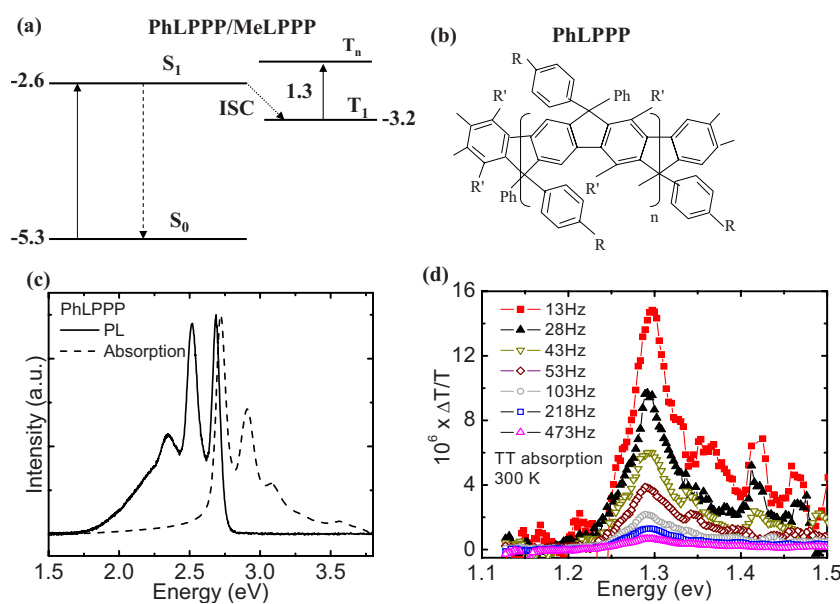

FIG. 1. (Color online) (a) Energy level diagram of PhLPPP/MeLPPP. (b) Chemical structure of PhLPPP. (c) PL and absorption spectra of a PhLPPP film. (d) Photomodulation spectra of PhLPPP at $300 \mathrm{~K}$ at different chopper frequencies; the peak at $1.3 \mathrm{eV}$ is the TT absorption.

2400 source meter. The external quantum efficiency (EQE) spectra were measured using a monochromatic light source from a Shimadzu RF-5301PC spectrofluorophotometer, a SR810 lock-in amplifier, and a Newport 918 Si photodetector for monochromatic power-density calibration. Optical studies from pristine and blended films spin-coated on quartz substrates with the same concentration and spin speeds as used in the PV devices were performed. The signature of the triplet-triplet (TT) absorption was measured from PhLPPP films using photoinduced absorption techniques. ${ }^{16}$

Figures 1(a) and 1(b) show the energy levels in PhLPPP and the chemical structure, respectively. Figure 1(c) shows the PL and absorption spectra from a PhLPPP film, which is very similar to that of MeLPPP. Continuous wave PL is not an adequate tool to observe phosphorescence in polymers where only trace quantities of heavy metal are introduced; gated detection techniques are required to observe the phosphorescence. ${ }^{15}$ Figure $1(\mathrm{~d})$ shows the $T_{1} \rightarrow T_{N}$ TT absorption peak at $300 \mathrm{~K}$ for the PhLPPP sample at different chopper frequencies. The TT absorption peak in MeLPPP, unlike PhLPPP, is only observed at low temperatures. ${ }^{16}$ By fitting the intensity of the TT absorption peak as a function of the chopper frequency $(f) \Delta T / T=C / \sqrt{1+(2 \pi f \tau)^{2}}$, where $C$ is a constant, we estimate the lifetime $(\tau)$ of the triplet excitons as $8.7 \pm 0.4 \mathrm{~ms}$ at $300 \mathrm{~K}$. The energy levels of PCBM (lowest unoccupied molecular orbital is at $-4.3 \mathrm{eV}$ ) match well with PhLPPP for the transfer of electrons from the singlet and triplet manifolds of PhLPPP to PCBM.

Figure 2 shows the current density-voltage $(J-V)$ characteristics of the $2: 1$ and $1: 1$ blend solar cells obtained under
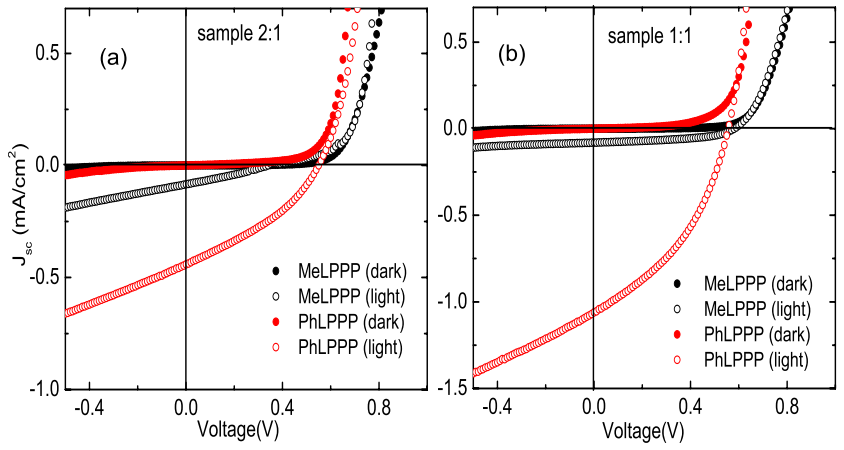

FIG. 2. (Color online) (a) Current density vs voltage curves for 2:1 MeLPPP:PCBM and PhLPPP:PCBM devices. Both the dark current density and the current density under simulated AM1.5 solar irradiation are shown. (b) Current density vs voltage (both dark and light) curves for 1:1 MeLPPP:PCBM and PhLPPP:PCBM devices.

dark and simulated AM 1.5 G illumination conditions. These results are an average of more than 100 devices that were fabricated using each of the blends. The bandgap of MeLPPP is $\sim 2.5 \mathrm{eV}$, which is far from the ideal low bandgap required for high efficiency polymer-based solar cell applications. Hence it is not surprising that the short circuit current density $\left(J_{\mathrm{sc}}\right)$ is smaller than what is reported for materials such as P3HT-based solar cells. ${ }^{9}$ Furthermore, the hole mobilities in PhLPPP and MeLPPP based polymers are a few orders of magnitude smaller than the electron mobilities in PCBM. The P3HT-PCBM system has similar mobilities for electrons and holes ensuring a balanced transport, and thus shows one of the highest power conversion efficiencies among polymer solar cells.

$J_{\text {sc }}$ is systematically lower for MeLPPP compared to PhLPPP blended solar cells as seen in Fig. 2 indicating the role of the triplet excitons in the latter. The 2:1 blended PhLPPP sample shows low fill factor $(\mathrm{FF})=0.34$ and power conversion efficiency (PCE) of less than $0.1 \%$. This is substantially enhanced in the $1: 1$ and 1:2 samples. The $1: 1$ and 1:2 samples under $100 \mathrm{~mW} / \mathrm{cm}^{2}$ illumination show an average power conversion ratio of $\sim 0.23 \%$. The FF and $J_{\mathrm{sc}}$ values of all devices are shown in Table I. The power conversion efficiency values in MeLPPP are reduced by a factor of 3-10 compared to the triplet-enhanced PhLPPP solar cell devices.

Figure 3(a) shows the EQE of the 1:1 PhLPPP and MeLPPP devices in the 300-650 nm range. The EQE of the $\mathrm{PhLPPP}$ device in the blue-green region is higher by a factor of 7-8 compared to the MeLPPP device. The inset shows the absorption spectra of the 1:1 blend for both polymers. Although the $0-0$ vibronic peak in the PhLPPP blend shows a slightly higher absorption coefficient compared to MeLPPP,

TABLE I. Summary of the performance of diodes fabricated using MeLPPP and PhLPPP. The last four columns denote the FF, open circuit voltage, short-circuit current density, and the PCE.

\begin{tabular}{lcccccc}
\hline \hline & $\begin{array}{c}\text { PCBM concentration } \\
(\mathrm{wt} \%)\end{array}$ & $\begin{array}{c}\text { Thickness } \\
(\mathrm{nm})\end{array}$ & $\mathrm{FF}$ & $\begin{array}{c}V_{\mathrm{oc}} \\
(\mathrm{V})\end{array}$ & $\begin{array}{c}\left(J_{\mathrm{sc}}\right) \\
\left(\mathrm{mA} / \mathrm{cm}^{2}\right)\end{array}$ & PCE $(\%)$ \\
\hline Mevice with active layer & & & 0.29 & 0.35 & 0.085 & 0.008 \\
PhLPPP/PCBM (2:1) & 33 & $80-90$ & 0.35 & 0.55 & 0.44 & 0.09 \\
MeLPPP/PCBM(1:1) & & & 0.46 & 0.57 & 0.08 & 0.02 \\
PhLPPP/PCBM (1:1) & 50 & $50-60$ & 0.41 & 0.55 & 1.05 & 0.23 \\
MeLPPP/PCBM(1:2) & & & 0.34 & 0.63 & 0.33 & 0.07 \\
PhLPPP/PCBM (1:2) & 67 & $80-90$ & 0.37 & 0.59 & 1.07 & 0.23 \\
\hline \hline
\end{tabular}



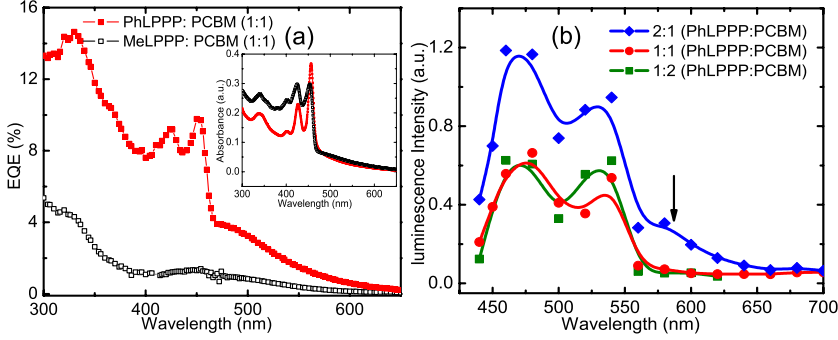

FIG. 3. (Color online) (a) EQE wavelength dependence of solar cells with PhLPPP:PCBM (1:1) and MeLPPP:PCMB (1:1) as active layers. The inset shows the absorption spectra of PhLPPP:PCBM (1:1) and MeLPPP:PCMB (1:1) films. (b) Luminescence from PhLPPP:PCBM blended 2:1, 1:1, and 1:2 films measured in air with a $9 \mathrm{~ns}$ pulsed source. The symbols denote the measured luminescence intensity at various wavelengths. The phosphorescence peak (at $\sim 590 \mathrm{~nm}$ ) denoted by the arrow almost disappears for the $1: 1$ and $1: 2$ blended films.

the overall absorbance of the two blends are comparable. Since the triplet excitons are being created due to an intersystem crossing-over from the singlet states, and then further disassociate, the EQE dependence on the wavelength will be similar to the singlet absorption. A recent work on an iridium incorporated polyfluorene shows a similar behavior. ${ }^{17}$

The PL spectra of the pristine polymers and the corresponding blends were also measured. The MeLPPP:PCBM samples demonstrate a slightly higher PL quenching compared to the PhLPPP:PCBM samples for the 1:1 and 1:2 blends. Additionally, the morphology of the PhLPPP:PCBM and MeLPPP:PCBM blends are similar as inferred from atomic force microscopy images of the blended films (not shown here). The device performance for the 1:1 PhLPPP and MeLPPP diodes are similar; by fits to the Schottky equation $I=I_{S}[\exp (e V / n k T)-1]$, the diode ideality factor $n$ is determined to be four for both. They both deviate from being ideal diodes but since the values are similar, the impact of the actual device performance on the PV efficiency is similar in both PhLPPP and MeLPPP. Thus the enhanced power efficiency and EQE in PhLPPP PVs must be attributed to the presence of mobile, longer living triplet excitons. The increased lifetime of the triplet excitons most likely increases the probability of finding nearby heterojunctions, where they dissociate and thus contribute to the photocurrent. To further understand the differences in the efficiency of the various PhLPPP:PCBM blended PVs, the luminescence decay pumped by a $Q$-switched Nd: YAG laser at $266 \mathrm{~nm}$ (fourth harmonic) with a pulse duration of $9 \mathrm{~ns}$ at a repetition rate of $5 \mathrm{~Hz}$ was measured from the blended films spin-coated on quartz substrates.

Figure 3(b) shows the luminescence intensity at different wavelengths measured from PhLPPP:PCBM blended films using the luminescence decay setup. The broad peak between $450-550 \mathrm{~nm}$ in the delayed luminescence has been attributed to emissive and oxidatively formed keto defects in PhLPPP. ${ }^{15}$ Since our measurements were performed in air, this broad peak masks the phosphorescence signal which is seen only as a shoulder around $590 \mathrm{~nm}$ marked by the black arrow. The defect emission is further reduced in the 1:1 and 1:2 blends, and the phosphorescence at $590 \mathrm{~nm}$ almost disappears in these films. These results correlate very well with the power efficiency of the PhLPPP:PCBM solar cells. For the triplet excitons to effectively contribute to charges, their radiative decay (phosphorescence) is undesirable. The 1:1 and 1:2 blended samples have the highest power conversion efficiency in good agreement with the delayed luminescence results, which show negligible phosphorescence (shoulder at $590 \mathrm{~nm}$ ) in these samples. Since the measurement times for all three samples were similar, the quenching of triplet excitons in the presence of oxygen is similar. Although the delayed luminescence measurements of the blended films were performed in air, the solar cells were encapsulated in an inert atmosphere; during the current-voltage measurements the polymer layers were not exposed to any air/oxygen ensuring that the triplet excitons were not quenched in devices.

We point out that simply mixing trace quantity of Pd complexes to MeLPPP does not necessarily work since the metal atoms may be far away from the polymer chain and may cluster. A side chain sequence during the polymer synthesis, as in PhLPPP, ensures the metal atoms to be covalently bound to the polymer backbone. Moreover, our results indicate that PV efficiencies may be enhanced even in large bandgap polymers by the contribution of triplet excitons.

The possibility of a triplet-enhanced power conversion efficiency in solution-processed LPPP-based ladder polymer:PCBM organic solar cells of the bulk heterojunction-type will stimulate further work on the development of lower bandgap conjugated copolymers with trace quantities of heavy metal. Since the contribution of the triplet excitons is weakly dependent on the morphology of the polymer and the resulting transport behavior due to their increased diffusion lengths, we anticipate that such a simple approach will be beneficial for improving power conversion efficiencies, especially in solution-processes or printed active layers without post-processing treatments that inherently have low-to-moderate PV efficiency.

This research was partially supported by grants from the U.S. National Science Foundation (Grant No. ECCS0823563) and the U.S. Army.

${ }^{1}$ Y. Shao and Y. Yang, Adv. Mater. (Weinheim, Ger.) 17, 2841 (2005)

${ }^{2}$ J. Peet, J. Y. Kim, N. E. Coates, W. L. Ma, D. Moses, A. J. Heeger, and G. C. Bazan, Nature Mater. 6, 497 (2007).

${ }^{3}$ D. Mühlbacher, M. Scharber, M. Morana, Z. Zhu, D. Waller, R. Gaudiana, and C. Brabec, Adv. Mater. (Weinheim, Ger.) 18, 2884 (2006).

${ }^{4}$ W.-Y. Wong, X.-Z. Wang, Z. He, A. B. Djurišiæ, C.-T. Yip, K.-Y. Cheung, H. Wang, C. S. K. Mak, and W.-K. Chan, Nature Mater. 6, 521 (2007).

${ }^{5}$ F. Guo, Y.-Gi. Kim, J. R. Reynolds, and K. S. Schanze, Chem. Commun. (Cambridge) 2006, 1887.

${ }^{6}$ J. Xue, S. Uchida, B. R. Rand, and S. R. Forrest, Appl. Phys. Lett. 85 5757 (2004)

${ }^{7}$ T. Kietzke, D. Neher, K. Landfester, R. Montenegro, R. Güntner, and U. Scherf, Nature Mater. 2, 408 (2003).

${ }^{8}$ G. Li, V. Shrotriya, J. Huang, Y. Yao, T. Moriarty, K. Emery, and Y. Yang, Nature Mater. 4, 864 (2005).

${ }^{9}$ M. Reyes-Reyes, K. Kim, and D. L. Carroll, Appl. Phys. Lett. 87, 083506 (2005).

${ }^{10}$ M. C. Scharber, D. Mühlbacher, M. Koppe, P. Denk, C. Waldauf, A. J. Heeger, and C. J. Brabec, Adv. Mater. (Weinheim, Ger.) 18, 789 (2006).

${ }^{11}$ Z. Xu, Y. Wu, and B. Hu, Appl. Phys. Lett. 89, 13111 (2007).

${ }^{12}$ J. S. Wilson, A. S. Dhoot, A. J. A. B. Seeley, M. S. Khan, A. Köhler, and R. H. Friend, Nature (London) 413, 828 (2001).

${ }^{13}$ A. Köhler and D. Beljonne, Adv. Funct. Mater. 14, 11 (2004).

${ }^{14}$ M. Reufer, P. G. Lagoudakis, M. J. Walter, J. M. Lupton, J. Feldman, and U. Scherf, Phys. Rev. B 74, 241201(R) (2006).

${ }^{15}$ J. M. Lupton, A. Pogantsch, T. Piok, E. J. W. List, S. Patil, and U. Scherf, Phys. Rev. Lett. 89, 167401 (2002).

${ }^{16}$ S. Yang, W. Graupner, S. Guha, P. Puschnig, C. Martin, H. R. Chandrasekhar, M. Chandrasekhar, G. Leising, and C. Ambrosch-Draxl, Phys. Rev. Lett. 85, 2388 (2000).

${ }^{17}$ G. L. Schulz and S. Holdcroft, Chem. Mater. 20, 5351 (2008). 